\title{
Research on the Aesthetic Value of Weinan Shadow Playing Music
}

\author{
Qian Wang
}

Xianyang Normal University, Shaanxi, Xianyang, 712000

Keywords: Weinan Shadow; Music Aesthetic Attributes; Aesthetic Value

\begin{abstract}
At present, the state for the shadow art and culture of the protection and inheritance efforts to increase, and gradually attach importance to the protection of the shadow play, in the development of outstanding culture of folk background, shadow play as the earliest and most complete drama art and pure folk rock music, This unique Chinese singing and dancing with the shadow of the art, began to be protected and inherited up, Weinan shadow play as one of the representative of the shadow play, to develop. Based on this background, this article based on the the development status quo of Weinan shadow play, research on the aesthetic value of its music and analysis the specific aesthetic attributes of music and music aesthetic value.
\end{abstract}

\section{Introduction}

Chinese all kinds of opera, all originated in the Shaanxi Qin cavity, the original Shaanxi originated in the shadow, shadow originated in Weinan, which can be said, Weinan shadow is the total source of all Chinese opera art. Weinan shadow play began to appear in the Western Han Dynasty, spread so far for more than two thousand years and its popular in the Tang and Song dynasties, in the Ming and Qing Dynasties reached its peak, itself with a long cultural and artistic atmosphere and traditional cultural aesthetic value. Weinan shadow play in 2006 by the Ministry of Culture included in the "first batch of national intangible cultural heritage protection list", in 2011 selected "human intangible cultural heritage representative list." At the same time, in the development of their own repertoire show a wide range of features, the introduction of martial arts drama, the history of traditional drama, children's drama and the main plane play and other play to meet the aesthetic needs of different audiences, thereby enhancing their aesthetic value.

\section{The Music Aesthetic Attributes of Weinan Shadow Play}

The Shadow Play Music Form Is With Agility. Weinan shadow opera singing inherited the traditional singing of the Qin cavity, and then evolved into time, in Weinan shadow play, mainly bowl cavity mainly in addition to the old cavity, cavity and other cavity development. As a whole, the musical form of the shadow play shows a wide range of development trends, from the multi-singing in the United States to give the experience of the United States, mainly because of suona as accompaniment instruments, it is also known as "blowing cavity." One for the four-sentence body, "from the inheritance" structure, artists called "flat (slow)", plus gongs and drums called "water"; the other is repeated up and down sentence singing, artists called "Stacking plate ". Bowl bowl because of a bowl of copper bells involved in accompaniment named, is a special board cavity structure cannot be conventional eye to regulate the beat. So that the overall shape of the singing in the singing reflects the flexibility, that is, aura full of aura, the shadow play interpretation of the fresh up, at the same time Weinan shadow play the melody tune clear, can be in the sentence and help the words are dynamic , From these two aspects reflect the aesthetic attributes 
of music. In the Qin cavity, the string and the board as the main features, especially in the bowls of bowls, because the bowl of the cavity for the shadow of the specific performance can be gentle and delicate, with a strong singing his voice to a story and a situation, singing rich Personalized and distinctive sense of spirit, will be different roles live. From the auditory aspects of the analysis, bowls of cavity delicate, able to change the sense of euphemism, true and false sound, and true and false voice to create the actual situation.

Shadow Play Music Performance Is Resonant. Weinan shadow play music performance is through the "five busy" way to show the whole shadow play, including the lead singer's "before the sound", and play drums on the moon, at home this need to show that the shadow play in a variety of different characters Complex feelings, in order to directly with the audience to produce emotional echo; responsible for manipulating the shadow of the "sign"; responsible for knocking gong, playing bowl, hit the cymbals, playing Bangzi "saddle"; responsible for pulling the two strings and blowing suona " File "and is responsible for pulling the board $\mathrm{Hu}$, trombone and with the sign of the" down file ", you need to grasp the instrument tone, to meet the emotional requirements of the singing, thus forming a" singing, shadow, music, "three aspects of integration and unity , And then there is resonance. In the shadow play "Zixia Palace" in the "Zheng: This is Lvzuan Huan, petal children, where are you? Lv Zai Huan: poor children have not seen rice for two days. Wushou: pro-mother, who are they? Zheng: He is my ex-husband and children, because they are not a device, I am helpless and then ritual, here, now again wrapped around me, you teach how to say? "These different characters in the sentence, need to grasp the melody Change, adjust the tone of the instrument, and finally in the words of the characters, then the word on the need to sound in order to ensure that the character of the clear. In the "Shili Temple" in the two crying white plate, the whole crying and sad tone enveloped the whole word, from the music performance, people feel the heroine's grief and resentment. As a result of different emotional expression, so the audience produces emotion on the plot and the plot characters have emotional resonance.

The Musical Structure of the Shadow Play Is Fragmented. The music structure of the shadow play is mainly composed of singing sentences, supplemented by the cavity, including its singing style, singing melody and tuning plate road and so on, as a whole is to keep the singing as the center of the launch structure. But the shadow of the music structure is different from the pure music in time and space on the structure of integrity and coherence, in order to express the shadow play and the different scenarios, the general is the use of fragmented scattered structure, shadow opera singing structure and length, Must be decided by the shadow play and the story of the plot, according to the development of the plot for a variety of melodies and tunes and even "Huayin" and "crying" on the transformation. For example, in the "Wan Fulian" first out of the name, "Long as dry: (poem) since childhood has been learning saints, the article arrogant and the whole. Chun Lei soon fish dragon, elated toward the class." But this start situation, the first appeared in the three fragments, the first is the dragon like a dry melody sentence smooth, the second is singing in the "since childhood has been learning sage, articles armed with the whole" with the first fragment The tone of the melody is different, as the tight board, melody tone rise, the third fragment is put forward ambition, tone to achieve the highest, and the tone of the tune up. In addition, in the "ten king temple" in the heroine has a song need to cut into the first section of the tip, and then tighten the board into the second fragment, and then down the second bit of the second board of six "music show, this Is the three fragments based on different performance motives, making a musical form of intermittent reached three times, but it is naturally independent of the use of the jump and sound echoes the way to establish a unique music structure. 


\section{The Music Aesthetic Value Embodied in Weinan Shadow Play}

Enhance the Effect with Melody to Attract the Audience. Weinan shadow play the unique melody, the singing melody can be suitable for the audience to wear language and emotional changes in the tone. It is closer to the distance between the two sides, while the three-dimensional melody highlights the distinctive character, in the accompaniment of the instrument, sometimes elegant and sometimes angry, so that the audience obsessed. For example, "Spring and Autumn" tells the story of a pair of talented people after the hardships of the love story. In the plot of the young girl Jiang Qiulian mother died, his father came out to do business, often by the mother of the abuse of the plot to the boring bass to show the grievances of Jiang Qiulian heart and where the situation, causing the audience to Jiang Qiulian's mercy, to attract the attention of the audience. In the plot progress, Jiang Xiulian with the mother of the mountain to pick up the firewood, Lu Yung-hui Li Chunhua, Li sympathy ginger encounter silver help, ginger in the help of the love of Lee's love sentence, The heart of the cheerful and the girl's modesty, the plot was added to the male and female heroes of the initiation of the plot to enhance the main line of the audience to achieve the purpose of this direct acceptance of the emotions. In the last, Zhang Yan line posing as the court members rushed to the court, punished his mother, Hou Shangguan and county magistrate dry wicked, rescued the injustice of $\mathrm{Li}$ Chunhua, and on the spot to $\mathrm{Li}$, ginger two chapel married storyline performance, "Li Chunhua answer To the head of the low, "the authentic Xiangfu tune board, a chant three sigh, nine ileum, singing people intoxicated, with memorable charm, which is its unique melody changes, showing a unique of the artistic charm, so as to achieve the aesthetic requirements of the audience, thus forming a new aesthetic value. Its music system for the typical board cavity on behalf of, can express students. Dazzling array of shadow, so that the first close contact with the shadow of the reporter dizzying, amazing. Each of the various shapes of the shadow, are a total of delicate and meticulous, exquisite form, pattern elegant features, with a strong national cultural connotation and regional atmosphere to express the traditional Chinese folk art deep historical background.

Express the Story with Music and Cause Resonance. The whole music structure of the shadow play can be changed with the temporal and spatial changes of the narrative in the shadow play, and it is closely related to the different plot and characters. The music structure of the shadow play has the special direction, mainly to the storyline and the human psychological change. Together, thus enhancing the performance of music, embodied in two aspects, on the one hand is the music and character psychological fit, causing the audience to the psychological awareness of the characters, to achieve resonance. Shadow play music and the screen closely, with the screen content, follow the main line of the main feelings of the hero. For example, "Golden Bowl Chai" Cuihu "borrowed water" section, the instrument on the use of thirty-six Bangzi submarine board and a single treble drag cavity, the song shows the Cuihu the hero's inner frustration and then become open-minded psychological changes, the performance of the characters into the Taolin, the walk Taolin, enjoy the natural scenery of the story, from the music and the audience's aesthetic point of view and aesthetic fit, causing the audience resonance. On the other hand is because the music is as a shadow play and image composition of the image of an important part of the composition for the picture rendering atmosphere, teaser. From the relationship between music and shadow play, they are the relationship between the whole and part. No matter what kind of shadow play music, are to reflect the overall concept of the screen as the yardstick, and not a single effect or the effect of musical instruments as a standard effect. Its existence and development must conform to the concept and needs of the shadow play, and not from the picture of the overall tone, isolated emphasis on the integrity of the music and expressive force. For the viewer, the shadow play music 
is embodied in the specific space, and the contents of the singing and the instrument are more specific and clear. The audience is more likely to understand and accept the shadow of the music, calmly resonate.

Tune with the Plot and Render the Atmosphere. Weinan shadow play in the tune on the combination of the North of the heroic and Nanqu gentle, reflecting the sense of hierarchy, the flexibility to use these tunes, combined with different scenes using different tunes, the whole song of the song sometimes passionate sometimes lingering, Sad, sound blend, rendering the entire performance atmosphere, so as to achieve the aesthetic experience of hearing. At the same time the tone of the tone, tone, crying, the general mood with the tone, the performance was happy with the flower tone, the performance of passionate when used crying. For example, in the "white fine" in the life of Li Qingyan to Zhenjiang case Miss Shangfei Qiong, the two love at first sight, the dream of love in the paragraph, the tone to maintain a higher, showing a happy situation, rendering two love Atmosphere. It often "show", "retired", "selling water", "trapped", "messenger", "road", "sacrifice pile", which "table flower" there are flat tone, there are high and low tone, in addition to the use of the sharp tone, in the violent tone and then turn on the formation of high, combined with the specific plot to render a specific atmosphere.

\section{Conclusion}

Weinan shadow play music aesthetic value research is as an important part of the study of the aesthetic value of local characteristics of culture, but also to promote the development of Weinan shadow play a major content. Based on the analysis of the aesthetic attributes of Weinan shadow play, this paper deeply studies the aesthetic value of Weinan shadow play music. The aesthetic attributes of Weinan shadow play reflect the aesthetic value of the shadow play in Qinchuan area.

\section{References}

[1] Shi Shuai. A comparative study on the artistic styles of the old chamber shadow and time shadow [J] .Journal of Literature and Art Review, 2015, (05): 151-155.

[2] Ma Yaqin. Shaanxi east road shadow play aesthetic value[J]. Contemporary drama, 2011, (05): 36-38.

[3] Yang Shengbo, Wang Qing. Shaanxi shadow shadow, paper cutting, woodcut New Year value evaluation and protection [J]. Fine Arts, 2010, (11): 56-57.

[4] Yu Chaowu. Shaanxi east road shadow play [A]. Sanqin Culture Research Association Annual Record. 2008: 11

[5] Yang Junyan. On Weinan shadow play art [J]. Journal of Weinan Teachers College, 2008, (03): 50-52.

[6] Wang Xiaoguang, Qin Yongjun. Qing Dynasty shadow play box appeared Weinan [N]. Xinhua Daily Telegraph, 2002-12-17 (008).

[7] tribe left foot. Old cavity of the old[J]. Contemporary drama, 1989, (03): 62-63.

[8] Old chamber [J]. Shaanxi drama, 1960, (04): 15. 